\title{
NATURAL HISTORY OF THE DUCTUS ARTERIOSUS IN EXTREMELY LOW GESTATIONAL AGE NEONATES WITH MINIMAL INTERVENTION
}

\author{
C. Piyasena, J.A. Orme, E.D. Johnston, L. Psiouri, C. Kissack, B.J. Stenson \\ Simpson Centre for Reproductive Health, Royal Infirmary of Edinburgh, Edinburgh, UK
}

Background: Despite little evidence of benefit and significant potential for harm, patent ductus arteriosus (PDA) treatment is widespread. Trials comparing treatment with observation are absent. The rate of spontaneous closure in extremely low gestational age neonates (ELGANs) is unknown. We aimed to identify the spontaneous duct closure rate and clinical outcomes of a population of ELGANs when PDA treatment is rarely employed.

Methods: The outcomes of all infants, inborn or outborn before 28 weeks, and admitted on their day of birth to the NICU of the Simpson Centre for Reproductive Health, Edinburgh, during the 5 years 2006-2010 were ascertained. PDA treatment was recorded. Outcomes of infants with a murmur at discharge were ascertained.

Results: 163 ELGANs were admitted. 27 (16.6\%) died before discharge. No infant received COX inhibitors. 2 infants had surgical ligation of PDA before discharge. Of 136 infants discharged alive, 31 had a murmur at discharge. 1 of these had PDA treated with COX inhibitors and 1 surgically in other units. 1 infant had surgical closure in infancy after discharge home. 19 murmurs resolved on follow up. 7 infants still have murmurs on follow up. 2 infants have unknown status. Other outcomes were: grade III/IV IVH: 17 (10.4\%), NEC $\geq$ stage 2: 26 (16\%), Laser-treated ROP: 30/131 (22.9\%), BPD at 36 weeks in survivors 80/129 (62\%).

Conclusions: In a population of 163 ELGAN infants with $83.4 \%$ survival to discharge, spontaneous duct closure occurred eventually in $89.7 \%$. Trials of treatment versus observation are needed to determine whether treatment is advantageous. 